\title{
23 \\ Generation of conformance test suites for B-ISDN signalling relevant to Multi-party testing architecture
}

\author{
Jin Young Choi, Beom kee Hong \\ Electronics and Telecommunications Research Institute(ETRI) \\ 161 Kajong-Dong, Yusong-Gu, TAEJON, 305-350, KOREA \\ E-mail:jychoi@testopia.etri.re.kr,bkhong@testopia.etri.re.kr \\ TEL : +82-42-860-5858, FAX: +82-42-860-5440
}

\begin{abstract}
In this paper, we introduce the development experiences of conformance test suites for BISDN signalling protocol Q.2931. We developed Abstract Test Suites for the user and network side respectively, and made them executable by designing and realizing a test system. In our procedures, we applied not only the Single-party testing but the Multi-party testing for each test suite. Particularly in the Multi-party testing for the network side, we made the test suite by applying the method to control and observe two ports of the IUT concurrently. We also verified the test suites by internal testing with reference systems. These are able to increase the possibility of the interoperability among the systems implemented on different environments.
\end{abstract}

\section{Keywords}

Conformance Test, ATS, ETS, Multi-party testing, B-ISDN

\section{INTRODUCTION}

A communication protocol is a set of rules that defines all possible interactions among communicating systems. As communication protocols are more complicated and require higher reliability, it is necessary for an implementation to be checked for conformity to its standard specification to ensure that such implementations communicate reliably. This procedure is known as conformance testing(Sarikaya, 1989). 
Up to the present there have been some Abstract Test Suites(ATSs) for conformance testing of communication protocols such as X.25 used in packet network and Q.931 in NISDN. However, standardization activities for ATS of the B-ISDN protocol with which various multimedia services will be introduced are at an early stage. The ATS for B-ISDN protocol is not standardized yet except the ones for ATM layer and AAL protocol. This paper is concentrated on the generation of test suites for B-ISDN signalling protocol which defines call control procedures for end-to-end connection. Since this protocol is not symmetric for the user side and the network side, the ATS is needed for each side. The test methods considered for the development of the ATS should be also different for both cases. Particularly for the network side protocol, consideration of a different method rather than the Single-party testing which is used conventionally is needed, that is the Multi-party testing.

ISO/IEC 9646 deals with the conformance testing methodology, the development of ATS using Tree and Tabular Combined Notation(TTCN), the execution of test, the testing result analysis, and so on(ISO/IEC 9646-1 Information technology - Open System Interconnection Conformance testing methodology and framework - Part 1,2,3, 1994). There are two possible conformance testing architectures according to the test configuration: the Single-party testing architecture and the Multi-party testing architecture. The Single-party testing can be applied when an Implementation Under Test(IUT) is required, by the test purpose, to communicate with only one real other system. The Multi-party testing can be needed when an IUT is required, to communicate with multiple other real systems concurrently by means of the same or different service providers. In practice in order to perform conformance testing, it is necessary to select a proper testing architecture depending on testing environmental restrictions.

In this paper we describe the methodology and architectures that we have considered for the development of Q.2931 protocol's ATS and some procedures and techniques that we have taken during the development of ATS and the implementation of ETS. In particular we realized the ETS over the platform developed by ourselves and verified some conformance testing functions. This paper consists of as follows. We describe characteristics of B-ISDN signalling protocol in section 2 . In section 3 , we address testing architectures that we have adopted for conformance testing, and the ATSs for user side and network side of Q.2931 protocol are described in section 4 . Section 5 describes the implementation of ETS from the ATS. Finally, section 6 concludes the paper.

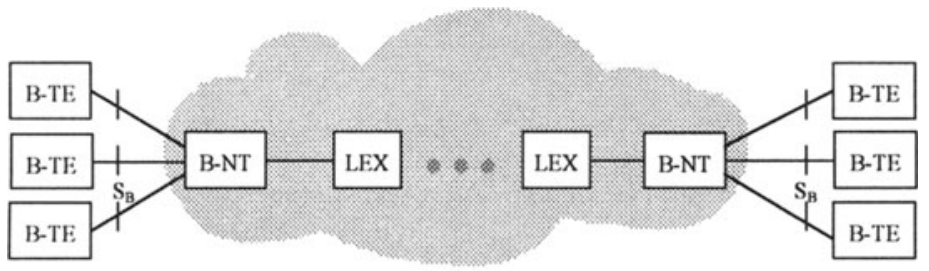

B-NT Network termination for B-ISDN

B-TE Terminal equipment for B-ISDN

LEX Local Exchange for B-ISDN

Figure 1 A reference configuration of B-ISDN. 


\section{CHARACTERISTICS OF B-ISDN SIGNALING PROTOCOL}

B-ISDN is a digital communication network that utilizes broadband transmission and switching technologies to interconnect the concentrated or distributed subscribers and the service providers, and to support integrated services with wide-bandwidth distribution. Figure 1 shows a reference configuration of B-ISDN. It contains the following:

\section{- Functional groups : B-NT, B-TE, and LEX}

- Reference points : $S_{B}$.

B-ISDN is composed of several protocols such as physical layer, ATM layer, AAL, and high layer for signalling, and each layer has its own functions. Among these protocols, Q.2931 protocol is a B-ISDN Digital Subscriber Signalling System No. 2(DSS2) User-Network Interface(UNI) layer 3 specification for basic call/connection control(ITU-T Draft Recommendation Q.2931, 1994). This protocol specifies the procedures for the establishing, maintaining and clearing of network connections at the B-ISDN user network interface. The procedures are defined in terms of messages exchanged and applied at the interface between a B-ISDN terminal and a B-ISDN public network(reference point $S_{B}$ and $T_{B}$ coincident) as well as at the interface between a B-ISDN customer network and a B-ISDN public network(i.e. at the $T_{B}$ reference point). The flow of messages for $Q .2931$ call/connection control is shown in Figure 2 .

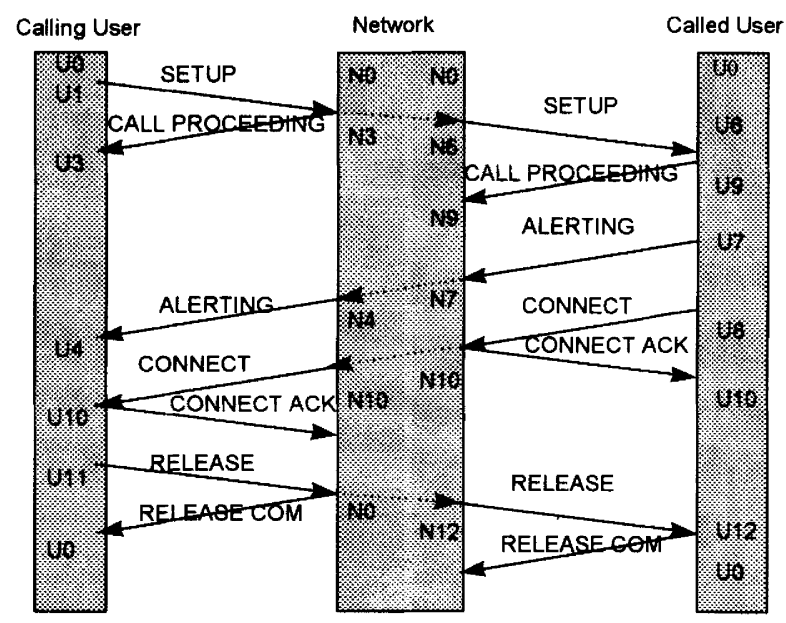

Figure 2 Flow of messages for Q.2931 call/connection control.

The main feature of this protocol is that the procedures for user side and network side are asymmetric. First of all, the behaviors of the calling user entity and the network entity of the destination interface are defined differently. Also the behaviors of the called user entity do not correspond with the behaviors of the network entity of the originating interface. For example, 
when the calling user sends a SETUP message to the network, the calling user enters the U1(Call Initiated) state. On the other hand, when the network sends a SETUP message to the called user, the network enters the N6(Call Present) state. In each case, the transition state and the reaction of the called user and the network are also different. A CALL PROCEEDING and ALERTING messages transmitted from the called user to the network as a response to the SETUP message are optional. Also some information elements included in messages are mandatory in the user-to-network or the network-to-user direction, but optional in the opposite direction. Therefore if we consider the conformance testing for Q.2931 protocol, it is obvious that a common test suite can not be applied for the user and network side. In particular, in case of the conformance testing of the network side, it is more meaningful to control and observe both interfaces of the network side simultaneously because in case of testing only one interface of the network side we should implement the UT function in the SUT or the called user system in the tester to play a role of corresponding counter part. Furthermore, the testing on the other interface should be performed again. On the other hand, if we control two interfaces in the tester at the same time, we can reduce those overloads and perform the testing more efficiently. This leads to consider a different testing architecture from the one of the user side. We describe the testing architectures adopted to the user and network side conformance testing in the next section.

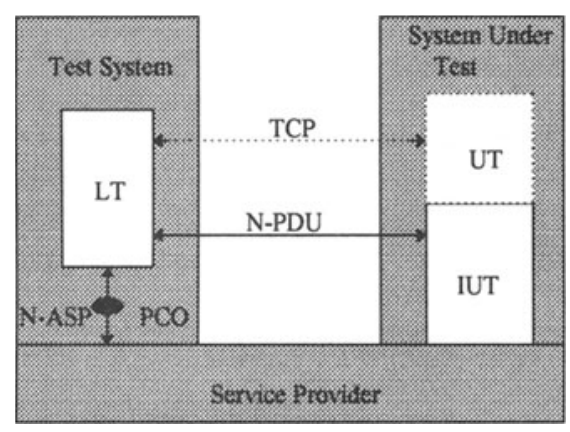

Figure 3 Applied Single-Party testing architecture.

\section{TESTING ARCHITECTURES}

In this section, we describe testing architectures considered for the development of Q.2931 user side and network side ATS(ISO/IEC 9646-1 Information technology - Open System Interconnection - Conformance testing methodology and frame work - Part 2, 1994).

\subsection{Single-party testing architecture}

We applied the Single-party testing architecture to the Q.2931 user side ATS configuration because it is sufficient to perform the test as exchanging messages between the test system and the IUT using only one connection. There are four methods in this architecture, and we 
decided to adopt Remote test method shown in Figure 3 as it could be applied to various systems flexibly manufactured by different vendors. In this configuration, provision is made for the case where it is not possible to observe and control the upper service boundary of the IUT. The test events at the LT PCO are specified only in terms of N-Abstract Service Primitive(NASP), and N-Protocol Data Unit(N-PDU). Some requirements for Test Coordination Protocol(TCP) and UT may be implied or informally expressed in the ATS, but no assumption shall be made regarding their feasibility or realization. The Remote test method can reduce the overloads to implement additional software or hardware for testing in the SUT. The IUT represents the Q.2931 user side entity and the System Under Test(SUT) can be B-TE or the user side of B-NT system.

\subsection{Multi-party testing architecture}

In case of intermediate systems, multiple protocols behave at multiple ports simultaneously. Therefore the Single-party testing method which was often used previously cannot be applied anymore. For the testing of these systems, it is required that the test method shall control and observe multiple ports at the same time, which can be achieved by the Multi-party testing.

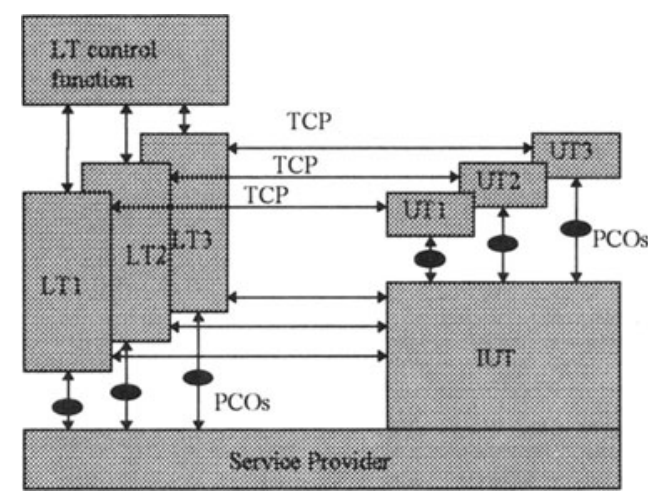

Figure 4 General Multi-Party testing architecture.

Q.2931 network side protocol running on an intermediate system behaves like those mentioned above by making a local call/connection. Therefore the testing must be performed as the procedure to stimulate an input to the IUT and to observe an output at the same port or other corresponding port. In case of Q.2931, since we consider only point-to-point configuration, it is sufficient to test only two ports in the IUT at the same time. Figure 4 shows the general architecture of the Multi-party testing, and Figure 5 is the applied testing architecture for the Q.2931 network side ATS configuration. This is also considering Remote test method. Two Lower Testers are located in the same test machine and the coordination function of those testers are more simplified. In this configuration, each LT represents one of the real systems with which the IUT needs to communicate. Each LT communicates with the appropriate part of the IUT, by observing and controlling ASPs and PDUs as in the Singleparty testing. The UT functions are not specified nor are any assumptions made regarding their 
feasibility or realization. This can reduce the testing overload on the SUT. The IUT represents the Q.2931 network side entity having more than two ports and the SUT can be the network side of B-NT system or switching system. The logical call/connection from LT1 to LT2 through SUT forms a local call/connection between two users. That is, if the IUT is the network side of B-NT system, LT1 and LT2 perform the roles of terminal equipment B-TE.

If we apply the Single-party testing to the Q.2931 network side ATS configuration, the following overloads would be forced for the testing of the network side of the IUT. Also we think that the Multi-party testing architecture like this could be extended to the interoperability testing among systems implemented on different environments.

- Implementation of the UT functions in the SUT

- Implementation of the called user system in the tester to play a role of corresponding counter part

- Increasing of the number of test cases by performing the test on the other interface of the network side.

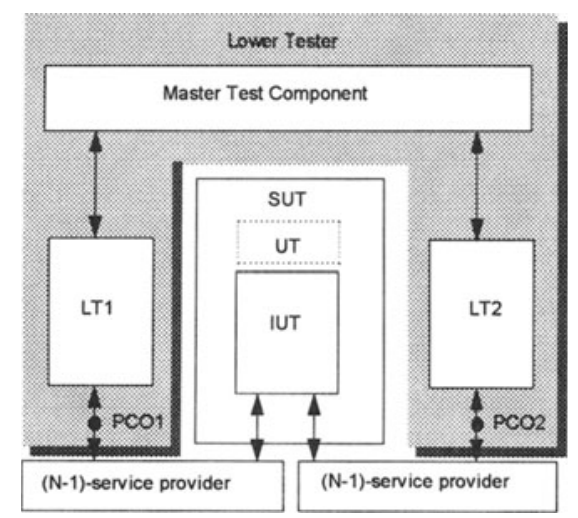

Figure 5 Applied Multi-party testing architecture.

\section{DEVELOPMENT OF ABSTRACT TEST SUITE(ATS)}

\subsection{Overview}

We developed the ATS for conformance testing using the following procedures. First of all, we made the Protocol Implementation Conformance Statement(PICS) proforma(ITU-T Draft Recommendation Q.931bis PICS and Abstract Test Suite for DSS1 Layer 3; Circuit Mode, Basic Call Control Conformance Testing, 1994). This is used for static conformance testing and composed of the following items.

- Major Capabilities

- Subsidiary Capabilities 
- Call States

- Supported Messages Received/Transmitted by the user(by the network)

- Message Structure

- Information Elements Received/Transmitted by the user(by the network)

- Supported Timers

- Additional Information for Interoperability Information Elements Structure.

Then we extracted the test purposes by analyzing Q.2931 specification. In this process, we generated the Extended Finite State Machine(EFSM) describing the procedures of the protocol Conformance Kit developed by Netherlands PTT was used as a development tool to get the raw level of the dynamic behavior part of the only user side ATS(Erik Kwast, Harma Wilts, Hans Kloosterman and Jan Kroon, 1991). But the results from the Conformance Kit did not resolve non-deterministic problems, timer operation and data flow parts of the protocol. So we made out test behaviors concerning these problems manually and the EFSM was only used as an input to the Conformance Kit. The test purpose document was used as the high-level test design document during the creation of tests in TTCN. We also made the state transition table based on the EFSM for the user side and network side ATS. This table represents an initial state, input/output event and a transition state. It also helps us extract test purposes from specification. Table 1 represents a part of the state transition table for the user side ATS. Each test purpose was expanded into detailed TTCN test behavior in the test suite. The writing of the test suite was a skilled manual activity.

Table 1 A part of the state transition table for the user side ATS

\begin{tabular}{|c|c|c|c|}
\hline State & $U O(N u l l)$ & $\begin{array}{l}\text { Ul(Call } \\
\text { initiated) }\end{array}$ & $\begin{array}{l}\text { U3(Outgoing Call } \\
\text { Proceeding) }\end{array}$ \\
\hline SETUP & $\begin{array}{l}\text { CALL_PROC, U9, } \\
\text { ALERTING, U7 or CONNECT, } \\
\text { Set T313=Now }+4, U 8\end{array}$ & $\begin{array}{l}\text { Ignore } \\
\text { U1 }\end{array}$ & $\begin{array}{l}\text { Ignore } \\
\text { U3 }\end{array}$ \\
\hline SETUP without MIE & $\begin{array}{l}\text { RELEASE_COM } \\
(\text { Cause }=\# 96), U 0\end{array}$ & - & - \\
\hline $\begin{array}{l}\text { SETUP with MIECE, } \\
(\text { IE flag }=0)\end{array}$ & $\begin{array}{l}\text { RELEASE_COM } \\
(\text { Cause }=\# 100), \mathrm{U} 0\end{array}$ & - & - \\
\hline $\begin{array}{l}\text { SETUP with UnrIE } \\
(\text { IE flag }=0)\end{array}$ & $\begin{array}{l}\text { STATUS(CS = U6,Cause }=\text { \#99) } \\
\text { CALL_PROC, U9, } \\
\text { ALERTING, U7 or } \\
\text { CONNECT, Set T313=Now } 4 \text {, U8 }\end{array}$ & - & - \\
\hline $\begin{array}{l}\text { SETUP with OIECE } \\
(\text { IE flag }=0 \text { ) }\end{array}$ & $\begin{array}{l}\text { STATUS }(C S=\text { U6, Cause }=\# 100) \\
\text { CALL_PROC, U9, } \\
\text { ALERTING, U7 or } \\
\text { CONNECT, Set T313=Now }+4 \text {, U8 }\end{array}$ & - & - \\
\hline
\end{tabular}


MIE : Mandatory Information Element

MIECE : Mandatory Information Element Content Error

UnrIE : Unrecognized Information element

OIECE : Optional Information Element Content Error

\subsection{User side ATS}

The ATS for conformance testing of Q.2931 user side protocol consists of Test suite overview part, Declarations part, Constraints part, and Dynamic part(ISO/IEC 9646-1 Information technology - Open System Interconnection - Conformance testing methodology and frame work - Part 3, 1994). In this test suite, PCO was declared only one, named L, because Remote test method considering the Single-party testing was applied.

In Test suite overview part, the structure of the test suite and test purposes were addressed. Declarations part defined some parameters, constants, variables, PDUs, and timers. A message, especially, has some Information Elements(IEs) and an IE also has some fields. Therefore the declaration of one message should have a structured type. For example, in Table 2 Connection Identifier IE in the ALERTING message was declared as a type CIdinfo substructure because it splits into some fields again. This scheme is also applied in Constraints part. This point is the main feature of Q.2931 messages compared to other protocol's PDUs below layer 2 and it forces more efforts to describe the ATS. Dynamic part of the test suite consists of behavior descriptions of test cases, preambles, postambles, and some test steps. The behavior of a test case was described as the procedure sending an input, receiving an output and verifying the transition state. We generated 397 test cases and these test cases can be divided into valid test cases, invalid test cases, and inopportune test cases.

Table 2 An example of PDU declaration

\begin{tabular}{|c|c|c|}
\hline \multicolumn{3}{|c|}{ PDU Type Declaration } \\
\hline \multicolumn{3}{|c|}{$\begin{array}{l}\text { PDU Name : ALT } \\
\text { PCO Type : LSAP } \\
\text { Comment : alerting, } \mathrm{u} \leftrightarrow \mathrm{n}\end{array}$} \\
\hline Field Name & Type & Comments \\
\hline$\overline{\mathrm{PD}}$ & OCTETSTRING[1] & protocol discriminator, $\mathrm{M}$ \\
\hline CR & CRinfo & call reference, $\mathrm{M}$, OCTETSTRING[4] \\
\hline MT & MTinfo & message type, M, OCTETSTRING[2] \\
\hline ML & MLinfo & message length, $M$, OCTETSTRING[2] \\
\hline CId & CIdinfo & connection identifier, O, OCTETSTRING[4..9] \\
\hline NId & NIdinfo & notification indicator, O, OCTETSTRING[5] \\
\hline
\end{tabular}

The test suite structure of the Q.2931 user side ATS is as follows.

- Active IUT testing(Call/Connection control procedures at the originating interface) - for each state such as Null state(U0), Call Initiated state(U1), Outgoing Call Proceeding state(U3), Call Delivered state(U4), and Release Request state(U11)

- valid : General, Call reference, Timer

- invalid : Mandatory IE, Optional IE 
- inopportune : Message sequences

- Passive IUT testing(Call/Connection control procedures at the destination interface)- for each state such as Null state(U0), Call Received state(U7), Connect Request state(U8), Incoming Call Proceeding state(U9), and Active state(U10)

- valid : General, Call reference, Timer

- invalid : Mandatory IE, Optional IE

- inopportune : Message sequences.

\subsection{Network side ATS}

The overall structure of the network side ATS is the same as the user side ATS. The description scheme of each part in TTCN is also similar. The differences between them are the state classification and the number of PCO. In the user side ATS, the state in the test suite corresponds to the state in the specification and the number of PCO was one. However in case of the network side ATS, there are some points to be considered more carefully.

As described in section 3.2, it is proper that conformance testing for Q.2931 network side requires a different testing method rather than the Single-party testing which is usually used. So we adopted the Multi-party testing composed of two LTs. In this configuration, the tester controls and observes two PCOs at the same time which correspond two ports of the IUT.

The most important activity is the creation of a set of test purposes which explains the proposed test method, architecture and gives a precise definition of the purpose of each test. Test purposes of the user side ATS were generated according to the call state defined in the specification. But in the network side, we considered two port states simultaneously and made 6 combined states by the reasons mentioned in section 3.2. Because two ports have the dependent relation and we should control two ports simultaneously, some combinations of two port states does not exist together at some time points. There are 11 call states in the network side protocol and theoretically there can be 121 combination states for two ports. But we reduced combination states to 6 combined states as follows by the above reasons. Each denotes the port 1 and port 2 state respectively and the port 1 is the originating side of a call/connection for convenience. Some states such as Call Initiated, Connect Request, and Release Request are not considered explicitly by the limitations of Remote test method and assumed an implicit state transition.

- Null(N0)/Null(N0)

- Outgoing Call Proceeding(N3)/Call Present(N6)

- Outgoing Call Proceeding(N3)/Incoming Call Proceeding(N9)

- Call Delivered(N4)/Call Received(N7)

- Active(N10)/Active(N10)

- $\quad$ ull(N0)/Release Indication(N12)

Table 3 represents an example of Dynamic part described under these considerations. We described events occurred at two PCOs sequentially considering the order of them in the ATS not using concurrent TTCN. This is due to the testing architecture used for and additionally this makes the implementation of the ETS easier. We generated 136 test cases for the network side ATS. The overall test suite structure is similar to the one of the user side ATS. It is 
divided into 6 state groups and each group has several subgroups such as valid, invalid, and inopportune test groups.

Table 3 An example of Dynamic part

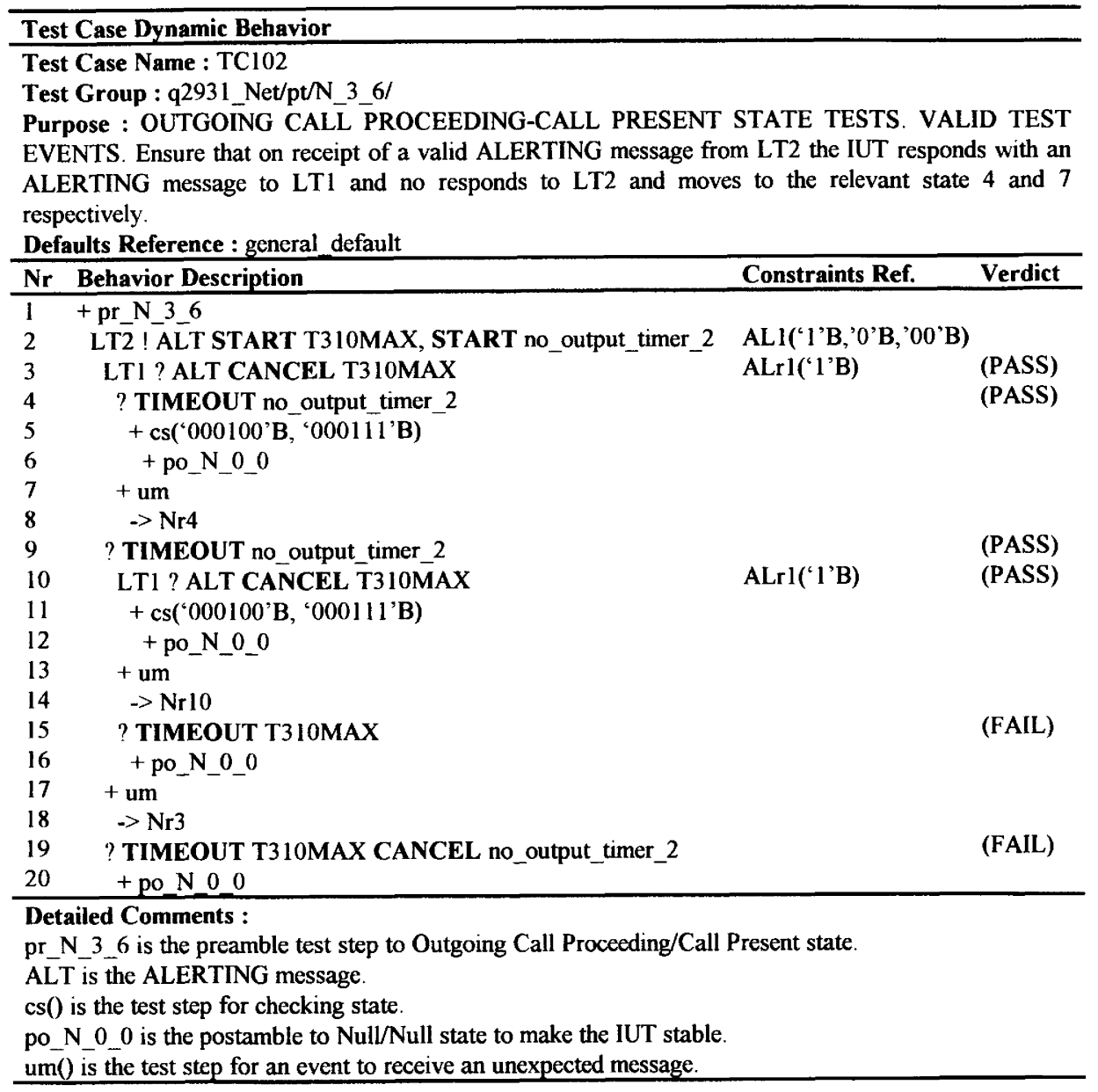

\section{IMPLEMENTATION OF EXECUTABLE TEST SUITE(ETS)}

\subsection{Test system design}

In order to realize the described test suites, we designed and developed the test system of 
which the block diagram is as Figure 6(K.Y.Kim, W.S.Kim, B.K.Hong, 1995). The system is composed of test management module, protocol testing module for two ports, low layer protocol processing module for each port, timer processing module, message distribution module, and IUT access module for each port. The functions of each module are as follows.

- test management module

It performs, controls, and manages the conformance testing.

- protocol testing module for two ports

It generates testing events for IUT and analyzes events received from IUT.

- low layer protocol processing module for each port

It performs emulating functions for low layer protocol and conveys testing events above and below.

- timer processing module

It processes information about timers.

- message distribution module

It sends testing events to the related port of IUT and delivers the received event to low layer protocol processing module of the corresponding port.

- IUT access module for each port

It provides the physical access point to the IUT. It consists of two ATM processing boards and one physical interface with two STM-1 ports.

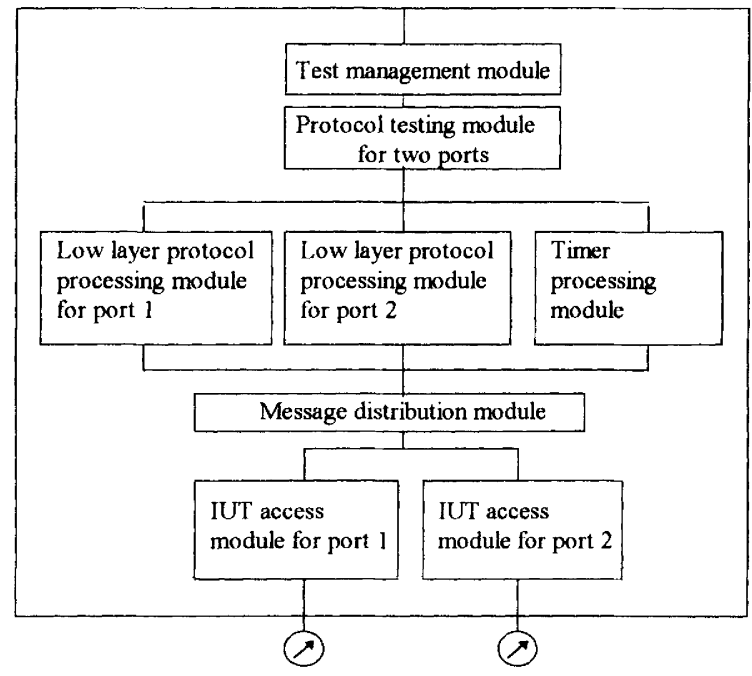

Figure 6 System block diagram.

Among these modules, the protocol testing module for two ports maintains information related each port. It also performs the role to exchange input/output with the related port through low layer protocol processing module and IUT access module for each port according to the testing purpose. Therefore in case of the Single-party testing of the user side ETS, only 
module of portl shall be used. Furthermore, for the IUT having more than two ports, the system could be extended as having multiple modules for them. The timer processing module solves timing problems by controlling all timers used in the test suite. It has timer values related to the execution of a test case and identifies each timer when a timer expires. Also because the Multi-party testing approach is used in the network side ATS, the coordination problem of LTs can be occurred. But two LTs are located in the same tester, the synchronization problem could be solved more simply. At each port the timer values of arriving events are observed and recorded in each event, and the timer is managed in the test system. So the protocol testing module can distinguish each event according to the order they arrive. By using this system, we realized the implemented ETS into the platform developed by ourselves.

Table 4 The constitution of the user side ETS

\begin{tabular}{lclc}
\hline \multicolumn{1}{c}{ Active IUT Test case } & \multicolumn{2}{c}{ Passive IUT Test case } \\
\hline & $\begin{array}{c}\text { number of } \\
\text { State }\end{array}$ & State & $\begin{array}{c}\text { mumber of } \\
\text { test cases }\end{array}$ \\
\hline Null(U0) & 1 & Null(U0) & 25 \\
Call Initiated(U1) & 53 & Call Received(U7) & 43 \\
Outgoing Call Proc.(U3) & 52 & Connect Request(U8) & 48 \\
Call Delivered(U4) & 47 & Incoming Call Proc.(U9) & 43 \\
Release Request(U11) & 42 & Active(U10) & 43 \\
\hline
\end{tabular}

\subsection{User and Network side ETS}

We implemented executable test suites from the ATS by using a TTCN to C translator and adding required API libraries such as encoding/decoding a message, sending/receiving a message, timer operation, managing test results, and so on. A public software called VINCE was modified as a platform performing Q.2931 simulation and low layer service providing functions.(Advanced Research Projects Agency(ARPA/CSTO) and the Naval Research Laboratory(NRL/CCS) VINCE version 1.2, 1994) Conformance testing functions of Q.2931 user side are divided into the passive IUT testing and the active IUT testing. The passive IUT testing is that the initiation of a test is caused by the tester. By triggering an input to the IUT a test case is started. In the active IUT testing, the IUT starts a test by sending an message(e.g. the SETUP message). This can be achieved by some user operation. Table 4 shows the constitution of the user side ETS implemented. On the other hand the network side ETS has two ports and the port corresponding to $\mathrm{PCO} 1$ is the originating side of a call/connection. But occasionally some test cases are started from PCO2. The distinction of the PCO for received messages is done by using the flag assigned by Message distribution module in Figure 6. Table 5 shows the constitution of the network side ETS implemented.

Functions implemented in the ETSs are as follows.

- declaration functions

They declare all information needed for initiation of testing.

- test case execution functions 
They execute the selected test case.

- test parameter input functions

They determine test parameters required to perform testing.

- sending message creation functions

They create message to be sent to the IUT.

- received message analysis functions

They analyze received messages and decide verdicts.

Table 5 The constitution of the network side ETS

\begin{tabular}{lcccccc}
\hline state & $N 0 / N O$ & $N 3 / N 6$ & $N 3 / N 9$ & $N 4 / N 7$ & $N 10 / N 10$ & $N 0 / N 12$ \\
\hline number of test cases & 25 & 27 & 30 & 28 & 12 & 14 \\
\hline
\end{tabular}

\subsection{Experiments with Test suites}

With these ETSs we needed to verify our test suites by removing errors, but there were no any formal methods. As an alternative, we decided to use any commercial or validated reference implementation. As a reference implementation, we utilized a public software called VINCE which was conformed to ATM Forum 3.1. But its functions are only for valid control flow parts and error handling procedures are not supported completely. Therefore we modified and supplemented the functions a little to be fitted to our specification of ITU-T Q.2931. Some features of error handling procedures were taken into account through these works.

In case of the verification of the user side ETS, we used the modified program directly. The program was connected to the test system with the ETS. All test cases were executed and the verdicts of pass or fail were generated. However we could not execute the network side ETS with the program as it was since the program did not have intermediate system functions. So we developed a simulating function working as an intermediate system and inserted it into the program. Similarly the program was connected to the system for two ports and all test cases were executed. Through these processes, we detected some faults in ATSs or ETSs and reflected them to corresponding suites.

We also applied the network side ETS to B-NT system that was developed by our institute. The testing scenario was as follows. The test system constituted a local call with B-NT system. Two LTs behaved like terminal equipment and were connected by B-NT through two ports. According to test procedures described in the ETS, two LTs stimulated inputs to B-NT and analyzed received outputs from B-NT. For each test case, the testing results were obtained. As a whole, the results of testing were on the good side.

\section{CONCLUSION}

Up to now we introduced our development procedures of conformance test suites for B-ISDN signalling. We developed the ATS for both the user side and the network side which was written by TTCN. In each case, we applied the Single-party testing and the Multi-party testing architecture respectively. Particularly in the Multi-party testing for the network side, we made out the test suite by applying the method to control and observe two ports of the IUT 
concurrently. In order to make the ATSs useful in the real world, we also realized ETSs in our test system. Through our verification procedures we detected some faults and reflected them correctly. The overall procedures described in this paper can be represented as Figure 7.

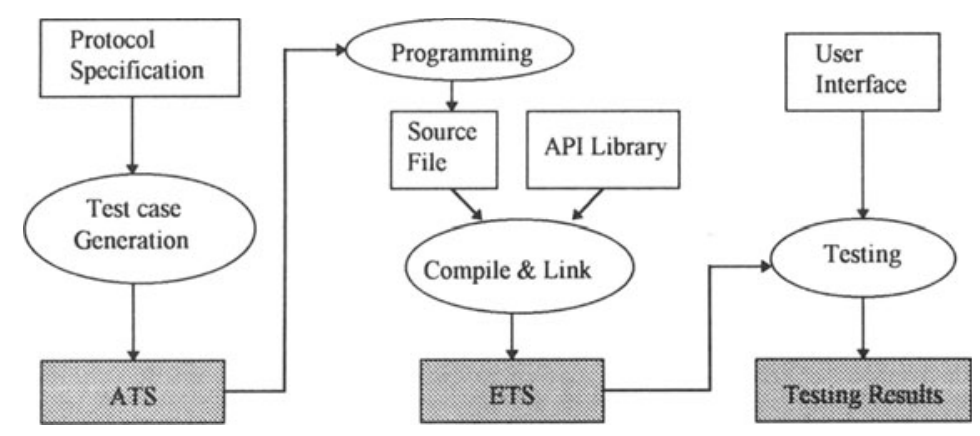

Figure 7 Overall testing procedures.

In the processes of developing the ATS and ETS, we had much skilled manual activities. In case of the ATS, we had to describe all definitions and constraints of messages relating to each test case. Though some definitions of messages were used commonly in several test cases, constraints were different for all test cases which had different test purposes. Particularly in the network side ATS, we had to consider all possible interaction between LTs through two ports for the description of the dynamic behavior part. We used a tool kit to implement the ETS, but it was necessary to provide API libraries supporting the ETS program at least.

With the testing environment mentioned in this paper, we have a plan to perform the conformance testing for the protocol implementation of B-TE, B-NT, ATM MSS, and switching system consisting of our B-ISDN testbed in the near future. We believe that these experiences can be applied to the development of test suites for more complicated protocols and they can increase the possibility of the compatibility and interoperability among the systems implemented on different environments.

\section{REFERENCES}

Advanced Research Projects Agency(ARPA/CSTO) and the Naval Research Laboratory(NRL/CCS). VINCE version 1.2. (1994)

Erik Kwast, Harma Wilts, Hans Kloosterman and Jan Kroon. (1991) User Manual of the Conformance Kit.

ISO/IEC 9646-1 Information technology - Open System Interconnection - Conformance testing methodology and framework - Part 1. (1994): General concepts.

ISO/IEC 9646-1 Information technology - Open System Interconnection - Conformance testing methodology and framework - Part 2. (1994) : Abstract Test Suite specification.

ISO/IEC 9646-1 Information technology - Open System Interconnection - Conformance testing methodology and framework - Part 3. (1994): The Tree and Tabular Combined 
Notation(TTCN).

ITU-T Draft Recommendation Q.2931. (1994) B-ISDN Digital Subscriber Signalling No. 2(DSS2) User Network Interface Layer 3 Specification for Basic Call/Connection Control. ITU-T Draft Recommendation Q.931bis PICS and Abstract Test Suite for DSS1 Layer 3; Circuit Mode, Basic Call Control Conformance Testing. (1994)

K.Y.Kim, W.S.Kim, B.K.Hong. (1995) Experience with the design of B-ISDN Integrated Test System. Proc. of IWPTS ' 95.

Sarikaya. (1989) Conformance Testing : Architectures and Test Sequences. Computer Networks and ISDN Systems, 17, 111-126.

\begin{abstract}
About the Authors:
Jin Young Choi is a member of engineering staff at the ETRI since 1993. He studied Industrial Engineering at the Hanyang University, Korea, and received a MS from the Korea Advanced Institute of Science and Technology in 1993. Current research interests include protocol conformance test, traffic of ATM network.
\end{abstract}

Beom Kee Hong is a senior member of engineering at the ETRI since 1982 and a project leader of B-ISDN protocol test. He studied computer science at the Hongik University, Korea, and received a MS from the Hongik University in 1984. Current research interests include protocol conformance test, ATM network testbed. 